\begin{tabular}{|c|l|}
\hline Title & $\begin{array}{l}\text { Growth of A IGaN GaN Quantum Wire Structures by Radio-Frequency-Radical-A ssisted Selective Molecular Beam } \\
\text { Epitaxy on Prepatterned Substrates }\end{array}$ \\
\hline Author(s) & Sato, Taketomo; Oikawa, Takeshi; Hasegawa, Hideki \\
\hline Citation & $\begin{array}{l}\text { Japanese Journal of A pplied Physics. Pt. 1, Regular papers, brief communications \& review papers, 44(4B), 2487-2491 } \\
\text { https://doi.org/10.1143/JAP.44.2487 }\end{array}$ \\
\hline Issue Date & 2005-04 \\
\hline Doc URL & http://hdl.handle.net/2115/33094 \\
\hline Rights & Copyright $\odot$ 2005 The Japan Society of A pplied Physics \\
\hline Type & article (author version) \\
\hline File Information & TSA TO17.PDF \\
\hline
\end{tabular}

Instructions for use 


\title{
Growth of AlGaN/GaN Quantum Wire Structures by Radio-Frequency-Radical-Assisted Selective Molecular Beam Epitaxy on Prepatterned Substrates
}

\author{
Taketomo Sato*, Takeshi Oikawa and Hideki Hasegawa \\ Research Center for Integrated Quantum Electronics (RCIQE) and \\ Graduate School of Information Science and Technology, Hokkaido University \\ North 13, West 8, Kita-ku, Sapporo 060-8628, Japan \\ *Corresponding author. E-mail address: taketomo@rciqe.hokudai.ac.jp
}

The feasibility of the selective molecular beam epitaxy (MBE) growth of AlGaN/GaN quantum wire (QWR) structures on prepatterned substrates is investigated. The detailed studies on growth features have revealed that size-reducing selective growth is possible on mesa patterns having the <11-20>-orientation, but not on those having the $<1-100>$-orientations. The behavior reflects complex growth kinetics on high-index crystalline facets. The lateral wire width of QWR structures formed selectively on a top mesa can be controlled by adjusting the growth thickness and the initial size of mesa patterns. From cathodoluminescence (CL) measurements, emission from the embedded AlGaN/GaN QWR structure has been clearly identified.

KEYWORDS: selective growth, molecular beam epitaxy (MBE), patterned substrates, GaN, $\mathrm{AlGaN}$

\section{Introduction}

Recently, the $\mathrm{AlGaN} / \mathrm{GaN}$ system has been opened up and has led to the establishment of blue/UV photonic and high-power electronic device application areas. This material system, however, is potentially suitable for not only these areas but also for high-operating-temperature quantum devices such as quantum-wire transistors (QWR-Trs) and single-electron transistors (SETs). This is due to wide energy gaps with a large $\Delta E_{\mathrm{c}}$ and to the availability of high-density two dimensional electron gases (2DEGs) even under non-doped conditions which avoids the doping fluctuation problem commonly observed in nanometer-scale devices. Here, a key question is how to form the AlGaN/GaN-based nanostructures in a size- and position-controlled manner. Recent studies on the fabrication of GaN-based quantum structures have been mostly focused on the self-assembled formation of quantum dots (QDs) using the Stranski-Krastanov mode [1,2] or anti-surfactant methods [3,4]. In these methods, control of size and position seems to be very difficult. On the other hand, we have shown that selective molecular beam epitaxy (MBE) growth on a prepatterned substrate is a very powerful approach for the formation of high-density quantum nanostructures for GaAs- and InP-based materials [5-7] with tight control of both position and size.

The purpose of this study is to investigate the basic behavior and feasibility of the selective $\mathrm{MBE}$ growth of $\mathrm{AlGaN} / \mathrm{GaN}$ quantum wire (QWR) structures on prepatterned substrates for the first time. For this purpose, $\mathrm{GaN}$ and $\mathrm{AlGaN}$ layers were grown by rf-radical-assisted MBE growth. Through growth on planar GaN (0001) substrates, the basic growth conditions were optimized using in situ reflection high-energy electron diffraction (RHEED) and X-ray diffraction (XRD) analyses. Then, selective growth experiments were 
carried out on patterned substrates. The structural and optical properties of the selectively grown $\mathrm{GaN}$ wires were characterized by scanning electron microscopy (SEM) and cathodoluminescence (CL) techniques. CL measurements were performed at $7 \mathrm{~K}$ using a cryostat.

A detailed study on the cross-sectional features of the grown structure has indicated that size-reducing selective growth is possible on mesa patterns having the $<11-20>$-orientation. The lateral wire width of QWR structures can be controlled by adjusting the growth thickness of the AlGaN and the initial size of mesa patterns. From cathodoluminescence (CL) measurements, the emission from the embedded $\mathrm{AlGaN} / \mathrm{GaN}$ QWR structures has been clearly identified.

\section{MBE Growth of GaN and AlGaN on Planar Substrates 2.1 Growth procedure}

In order to optimize the conditions for rf-radical-assisted MBE growth of GaN and AlGaN, uniform growth experiments were repeated on planar (0001) GaN substrates. Nitrogen was supplied using an rf radical source exited with a microwave power of $350 \mathrm{~W}$. In all experiments, the $\mathrm{N}_{2}$ flow rate and growth temperature, $T_{\text {sub }}$, were set to be $0.5 \mathrm{sccm}$ and $800^{\circ} \mathrm{C}$, respectively.

Prior to MBE growth, organic cleaning and HF treatment were applied in the atmosphere. Just before the MBE growth, thermal cleaning under $\mathrm{N}_{2}$ radical pressure was carried out in the MBE chamber for $5 \mathrm{~min}$. From detailed observations of RHEED patterns, cleaning at a substrate temperature, $T_{\text {sub }}$, around $800^{\circ} \mathrm{C}$ was found to be most effective in removing the native oxide or other contaminations from the surface.

\subsection{Results of uniform growth}

The measured growth rates of GaN and AlN layers are plotted in Fig. 1(a) as functions of the K-cell temperatures of the Ga source, $T_{\mathrm{Ga}}$, and $\mathrm{Al}$ source, $T_{\mathrm{Al}}$. The substrate temperature during growth was $T_{\text {sub }}=800^{\circ} \mathrm{C}$. The dashed lines in Fig. 1(a) show the expected group III limited rates of growth from the Arrhenius relationships for $\mathrm{Ga}$ and $\mathrm{Al}$ beam supplies from K-cells.

As for the growth of the $\mathrm{GaN}$ layer, the growth rate increased with $T_{\mathrm{Ga}}$ and showed saturation at around $T_{\mathrm{Ga}}=900^{\circ} \mathrm{C}$ with a rate of $100 \sim 150 \mathrm{~nm} / \mathrm{h}$, as seen in Fig. 1(a). This indicates that the growth became rate-limited by the V-group supply where the supply rate of Ga molecules became larger than that of nitrogen. In situ RHEED observation showed that well-defined bright streak (2x2) patterns, such as those shown in Fig. 1(b), were observed and maintained within the range of $T_{\mathrm{Ga}}=900 \sim 1000^{\circ} \mathrm{C}$. This result suggests that excess $\mathrm{Ga}$ adatoms on the growth surface easily desorb into vacuum, leading to the formation of a smooth surface even under the Ga-rich condition. On the other hand, the growth rate of the AlN layer did not show saturation over a wide range of $T_{\mathrm{Al}}=950^{\circ} \mathrm{C} \sim 1100^{\circ} \mathrm{C}$. However, at a high cell temperature $T_{\mathrm{Al}}$ of $1100^{\circ} \mathrm{C}$, the $(2 \times 2)$ RHEED pattern obtained at lower cell temperatures became dark and spotty, indicating the growth of an Al-rich poor-quality layer. From in situ RHEED observations, it was found that a growth rate within $100 \sim 150 \mathrm{~nm} / \mathrm{h}$ was suitable for the growth of both GaN and AlN layers.

The growth behavior of AlGaN layers is summarized in Fig. 2 where the incorporation rates of group III atoms into the planar (0001) epitaxial layer are plotted as functions of the Al atom supply rate. $T_{\text {sub }}$ was set to be $800^{\circ} \mathrm{C}$. The Ga supply rate was maintained at a constant value at $T_{\mathrm{Ga}}=940^{\circ} \mathrm{C}$. Here, the incorporation rates of group III atoms were determined by thickness measurements by cross-sectional SEM observation and Al composition measurements by XRD analyses. As seen in Fig. 2, the total amounts of Al and Ga adatoms incorporated into the surface remained constant in spite of the increase in Al supply rate, resulting in a corresponding decrease in the incorporation rate of $\mathrm{Ga}$ adatoms. This surprising 
result can be explained in terms of the group V supply limited growth in combination with the fact that the incorporation rate of $\mathrm{Al}$ adatoms is larger than that of $\mathrm{Ga}$ adatoms.

From the XRD analyses, it was found that the $\mathrm{Al}$ composition of $\mathrm{Al}_{\mathrm{x}} \mathrm{Ga}_{1-\mathrm{x}} \mathrm{N}$ layers could be changed from $x=0.24$ to 0.43 by changing the $\mathrm{Al}$ source temperature, $T_{\mathrm{Al}}$. A minimum FWHM value of the XRD peak of $300 \mathrm{~s}$ was obtained for both $\mathrm{GaN}$ and $\mathrm{Al}_{0.24} \mathrm{Ga}_{0.76} \mathrm{~N}$ layers grown at $T_{\mathrm{Ga}}=940^{\circ} \mathrm{C}$ and $T_{\mathrm{Al}}=980^{\circ} \mathrm{C}$, respectively. This value was equal to or even smaller than that of the MOVPE GaN template used in this study.

\section{Selective Growth of AlGaN/GaN Wire Structures 3.1 Growth method}

The sequence of selective growth is schematically shown in Fig. 3. As the template for selective growth, straight mesa stripes, as shown in Fig. 3(a) were formed by electron-beam (EB) lithography and etching on MOVPE (0001) GaN/ sapphire substrates. Since wet chemical etching is difficult for III-nitride materials, etching was carried out by an electron cyclotron resonance reactive ion beam etching (ECR-RIBE) process developed for nitrides by our group [8]. It uses a methane-based gas mixture of $\mathrm{CH}_{4} / \mathrm{H}_{2} / \mathrm{Ar} / \mathrm{N}_{2}=5 / 15 / 3 / 3 \mathrm{sccm}$ where the addition of $\mathrm{N}_{2}$ is extremely important. ECR plasma was exited at a microwave frequency of $2.45 \mathrm{GHz}$ with a power of $250 \mathrm{~W}$. In order to determine the suitable mesa orientations for the formation of quantum nanostructures, $<1-100\rangle$ and $\langle 11-20\rangle$ directions were investigated in this study, and the properties of selective growth for these mesa orientations were compared.

Then, selective growth of GaN-based QWR structures was attempted by depositing the materials shown in Fig. 3(b) onto the mesa-patterned substrate. First, a GaN buffer layer was grown on the patterned substrates. Then, an $\mathrm{Al}_{0.24} \mathrm{Ga}_{0.76} \mathrm{~N} / \mathrm{GaN} / \mathrm{Al}_{0.24} \mathrm{Ga}_{0.76} \mathrm{~N}$ sandwiched layer layers was deposited on the $\mathrm{GaN}$ buffer mesa, leading to the formation of embedded $\mathrm{GaN}$ wires, as schematically shown in Fig. 3(c).

\subsection{Growth selectivity on patterned substrates}

Cross-sectional SEM images of the $<1-100>$ - and $<11-20>-$ oriented mesa patterns fabricated by the ECR-RIBE etching process are shown in Figs. 4(a), 4(b) and 4(c). In this study, the etching depth of $450 \mathrm{~nm}$ was used for both orientations by controlling the etching time. After etching, well-defined crystalline facets appeared as sidewalls of the mesa stripe. As shown in Fig. 4(b), the mesa structures defined by two (11-22) side facets were obtained for the stripe in the $\langle 1-100\rangle$ direction. On the other hand, the mesa structures defined by two (1-101) facets were obtained in the $<11-20>$ direction, as shown in Fig. 4(c).

In order to investigate the feasibility of the selective growth of $\mathrm{QWRs}, \mathrm{Al}_{0.24} \mathrm{Ga}_{0.76} \mathrm{~N} / \mathrm{GaN}$ layers were grown on the mesa structures using the optimum growth conditions obtained using planar substrates. Figures 5(a) and 5(b) show the cross-sectional SEM images of the samples after the growth of $\mathrm{AlGaN}$ layer on $<1-100>$-oriented mesa structures with initial pattern sizes, $W_{0}$, of $750 \mathrm{~nm}$ and $200 \mathrm{~nm}$, respectively. The grown thickness was $300 \mathrm{~nm}$ in terms of growth on a planar (0001) GaN substrate. After the growth, mesa structures having a top width, $W$, of $760 \mathrm{~nm}$ and $200 \mathrm{~nm}$ were obtained, whose values were equal to or larger than the initial pattern width, $W_{0}$. Furthermore, the angle of side facets measured with respect to a (0001) plane slightly decreased after growth, as shown in Figs. 5(a) and 5(b).

On the other hand, it was clearly seen that the top width decreased after growth in the case of growth on the $<11-20>$-oriented mesa. Figures 6(a), 6(b) and 6(c) show the cross-sectional SEM images of the samples after the growth of AlGaN layers on the $<11-20>$-oriented mesa structures with initial pattern sizes, $W_{0}$, of $900 \mathrm{~nm}, 350 \mathrm{~nm}$ and $50 \mathrm{~nm}$, respectively. The mesa top widths, $W$, of $820 \mathrm{~nm}$ and $240 \mathrm{~nm}$ were obtained, respectively, for the samples with $W_{0}$ of $900 \mathrm{~nm}$ and $350 \mathrm{~nm}$. As for the sample with $\mathrm{W}_{0}$ of $50 \mathrm{~nm}$, a sharp 
ridge structure with $W$ below $10 \mathrm{~nm}$ was obtained after the growth. Furthermore, it was found that the angle of side facets remained constant at $62^{\circ}$, corresponding to the angle of (1-101) facets, during the growth for all samples, as seen in Figs. 6(a), 6(b) and 6(c).

The difference between the initial top width of mesa, $W_{0}$, and the top width of mesa after the growth, $W$, is plotted in Fig. 7 as a function of the grown thickness for two cases of growth on $<1-100>$ - and $<11-20>$-oriented mesa stripes. In the case of the growth of the GaN layer, $W$ decreased with growth thickness for both mesa orientations. The latter case indicates that the growth rate of $\mathrm{GaN}$ on the top facet is much larger than that on the side facet. This is very similar to the case of growth on GaAs (001) and (111)B patterned substrates [6].

Similarly, for growth of $\mathrm{AlGaN}$ on $<11-20>$-oriented mesas, the top width decreased as a function of growth thickness. On the other hand, in the case of the growth of $\mathrm{AlGaN}$ on $<1-100>$-oriented mesas, $W$ increased with growth thickness. This is probably because the growth rate on the side facets of the $\langle 1-100\rangle$-oriented mesa became larger than that on the top (0001). Such behavior could not be observed in the growth of GaN on the $<1-100>$-oriented mesas The present results indicate that, reflecting complex growth kinetics involving reaction and diffusion, incorporation rates of group-III adatoms are different depending on the adatom species, crystalline facets and growth conditions.

In order to further investigate how the wire width can be controlled, repeated wire growth experiments were carried out. Figure 8 shows the cross-sectional SEM image of AlGaN/GaN multilayers grown on the $<11-20>$-oriented mesa. The lateral width of GaN wires formed on the top facets was limited by two facet boundary planes (FBPs) which separate the growth region between the neighboring facets. The angle of FBPs, $\theta$, defined with respect to the (0001) plane is found to be $\theta=82^{\circ}$ according to Fig. 8. According to our previous paper [6], $\theta$ should satisfy

$$
\tan \theta=\frac{\alpha \cdot \tan \theta_{\text {side }}-\tan \theta_{\text {top }}}{\alpha-1}
$$

where $\theta_{\text {top }}$ and $\theta_{\text {side }}$ are the angle of the top facet and that of the side facet with respect to the top facet of the mesa pattern, respectively, and $\alpha$ is the ratio of the vertical growth rate on a top facet to that on a side facet. In the present case of the growth on $<11-20>$-oriented mesas, the values of $\theta_{\text {top }}=0^{\circ}$ and $\theta_{\text {side }}=62^{\circ}$ should be used as the angles of top (0001) facet and side (1-101) facets, respectively.

By using eq. (1) and the measured value of $\theta=82^{\circ}$, the growth selectivity, $\alpha$, is estimated to be 1.35. According to our model for the evolution of facet boundary planes, $W-W_{0}$ should be given by the following equation using the growth thickness on the top facet, $t$, and the boundary angle, $\theta$.

$$
W-W_{0}=-\frac{2 t}{\tan \theta}
$$

The lateral wire width geometrically estimated by this simple relation is also plotted in Fig. 7 for the growth of $\mathrm{AlGaN}$ on $<11-20>$-oriented mesas. It shows good agreements with the experimental data. Thus, the lateral wire width of a QWR structure formed selectively on the top of an $\mathrm{AlGaN}$ mesa can be controlled by adjusting the growth thickness and the initial size of mesa patterns.

\subsection{Selective growth of QWR structures}

On the basis of the above growth selectivity measurements, the selective growth of AlGaN/GaN QWR arrays was attempted on the $<11-20>$-oriented mesa substrates, using the material supply shown in Fig. 3(b). Figure 9 shows the CL spectra obtained from the wire 
region of the arrays of QWR structures. After the growth of the AlGaN/GaN/AlGaN sandwiched layer, the top width of the mesa was reduced, as expected, maintaining a smooth surface morphology, as seen in the inset of Fig. 9. As shown in Fig. 9, two sharp emissions were observed at $3.49 \mathrm{eV}$ and $3.69 \mathrm{eV}$, in addition to a broad yellow luminescence peak from $\mathrm{GaN}$ in the lower energy region.

The peak observed at $3.49 \mathrm{eV}$ comes from the band emission of GaN. Another peak observed at a higher energy of $3.69 \mathrm{eV}$ proves the successful formation of embedded $\mathrm{AlGaN} / \mathrm{GaN}$ QWR structures. However, evidence of lateral quantum confinements was not observed for the present sample. This is because the lateral wire width of the present wire structure, grown as a first attempt, is estimated from SEM observation to be $800 \mathrm{~nm}$, which is too large to expect a lateral confinement. The value of $3.69 \mathrm{eV}$ is consistent with the vertical confinement energy of $3.64 \mathrm{eV}$ that is estimated from the supply thickness of the GaN QWR layer. By further reduction of the initial mesa width, $W_{0}$, and by further optimization of growth conditions, it seems that narrower QWRs with strong lateral confinements can be realized.

\section{Conclusions}

In this study, the feasibility of the selective MBE growth of AlGaN/GaN QWR structures on prepatterned substrates was investigated. The main conclusions are listed below.

(1) In situ RHEED, XRD and SEM studies have revealed that the growth of GaN and $\mathrm{AlGaN}$ in the present study takes place in the group- $\mathrm{V}$ limited mode, while maintaining crystal quality similar to that of the MOVPE GaN template.

(2) The selective growth of $\mathrm{AlGaN} / \mathrm{GaN}$ QWR structures is possible on mesa patterns fabricated in a $<11-20>$ orientation where the lateral wire width formed selectively on the top facet of the mesa can be controlled by adjusting the growth thickness and the initial size of mesa patterns.

(3) From CL measurements, the emission from the embedded AlGaN/GaN QWR structure has been clearly identified. Further optimization of growth conditions should lead to the realization of QWRs with strong lateral confinements that are useful for electronic and photonic device applications.

\section{Acknowledgements}

The work reported here is supported in part by the 21st Century COE Project at Hokkaido University on "Meme-Media Technology Approach to the R\&D of Next-Generation Information Technologies" and by a Grant-in-Aid for Young Scientists (B)-16760239, both from the Ministry of Education, Culture, Sports, Science and Technology.

\section{References}

[1] M. Miyamura, K. Tachibana, T. Someya and Y. Arakawa: J. Cryst. Growth 237-239 (2002) 1316.

[2] J. Brown, F. Wu, P. M. Petroff and J. S. Speck: Appl. Phys. Lett. 84 (2004) 690.

[3] H. Hirayama, S. Tanaka, P. Ramvall and Y. Aoyagi: Appl. Phys. Lett. 72 (1998) 1736.

[4] S. Tanaka, M. Takeuchi and Y. Aoyagi: Jpn. J. Appl. Phys. 39 (2000) L831.

[5] H. Hasegawa, H. Fujikura and H. Okada: MRS Bulletin 24 (1999) 25.

[6] T. Sato, I. Tamai, S. Yoshida and H. Hasegwa: Appl. Surf. Sci. 234 (2004) 11.

[7] T. Sato, I. Tamai and H. Hasegawa: J. Vac. Sci. \& Technol. B 22 (2004) 2266.

[8] H. Hasegawa, T. Muranaka, S. Kasai and T. Hashizume: Jpn. J. Appl. Phys. 42 (2003) 2375. 


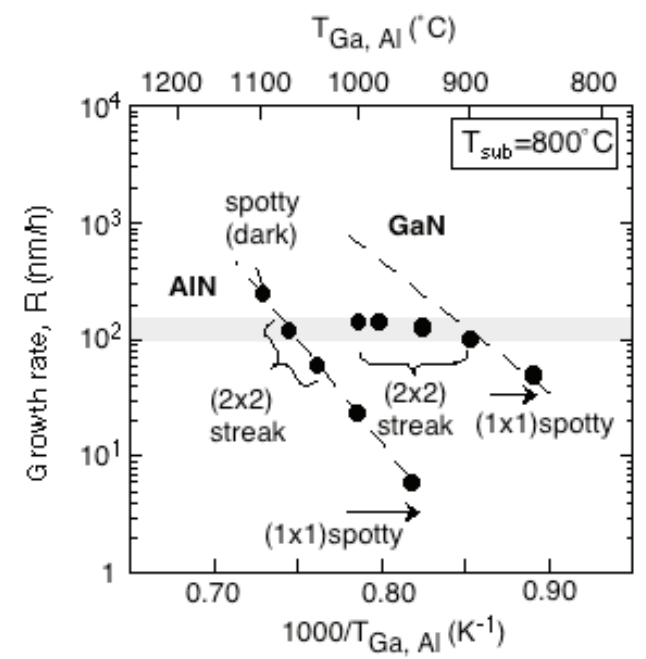

(a)

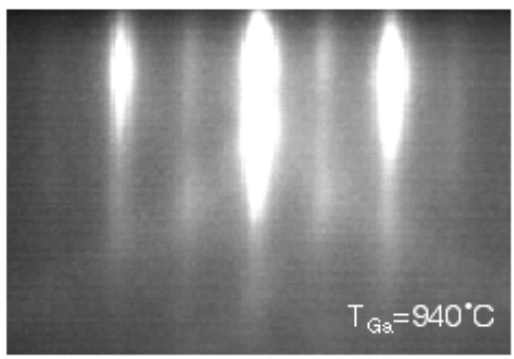

(b)

Fig. 1: (a) Growth rates of GaN and AlN and (b) (2x2) RHEED pattern obtained during $\mathrm{GaN}$ growth at $T_{\mathrm{Ga}}=940^{\circ} \mathrm{C}$.

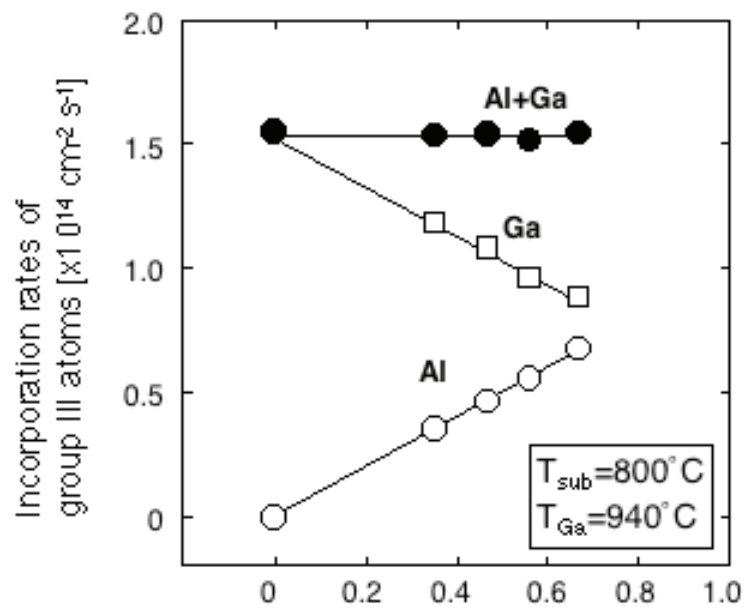

$\mathrm{Al}$ atom supply rate $\left[\times 10^{14} \mathrm{~cm}^{-2} \mathrm{~s}^{-1}\right]$

Fig.2: Incorporation rates of group-III atoms on planar substrates. The dashed lines indicate the growth rates expected from Arrhenius relationships of $\mathrm{Ga}$ and $\mathrm{Al}$ supplies from K-cells.

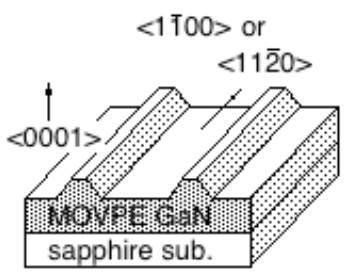

(a)

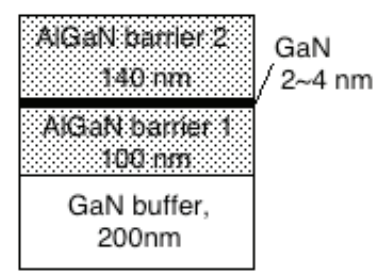

(b)

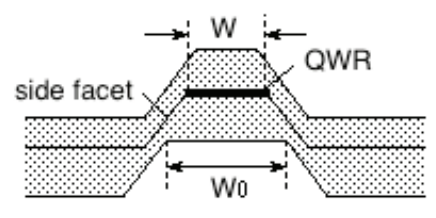

patterned sub.

(c)

Fig. 3: (a) Patterned substrate, (b) material supply and (c) schematic illustration of embedded QWR.

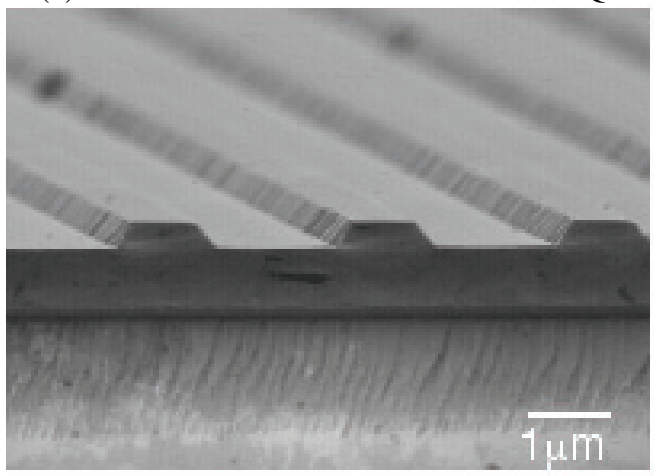

(a)

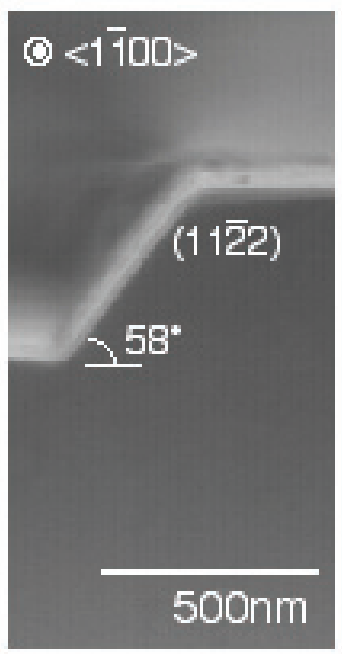

(b)

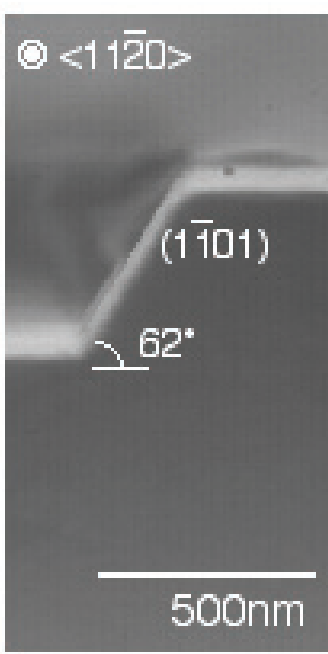

(c)
Fig. 4: Cross-sectional SEM images of patterned substrates fabricated by ECR-RIBE etching process. 


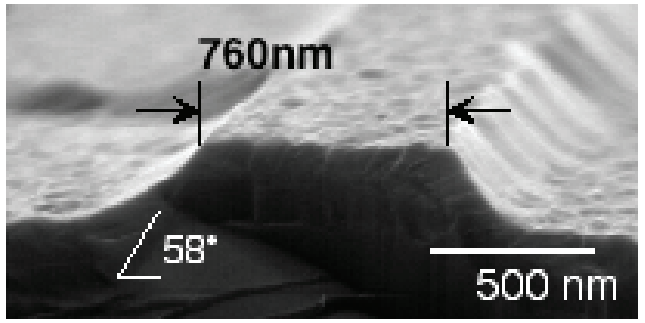

(a)

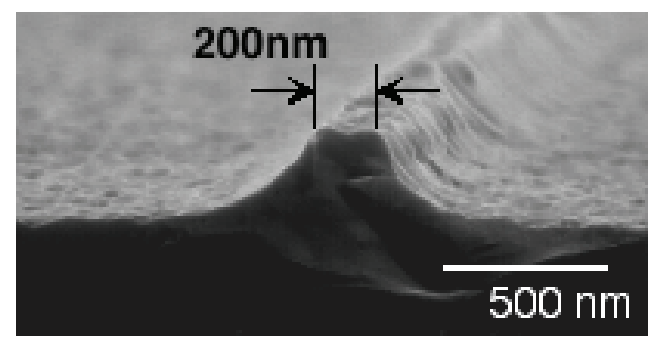

(b)

Fig. 5: Cross-sectional SEM images of samples after growth on $<1-100>$-oriented mesas having different pattern sizes of (a) $W_{0}=750 \mathrm{~nm}$ and (b) $W_{0}=200 \mathrm{~nm}$.

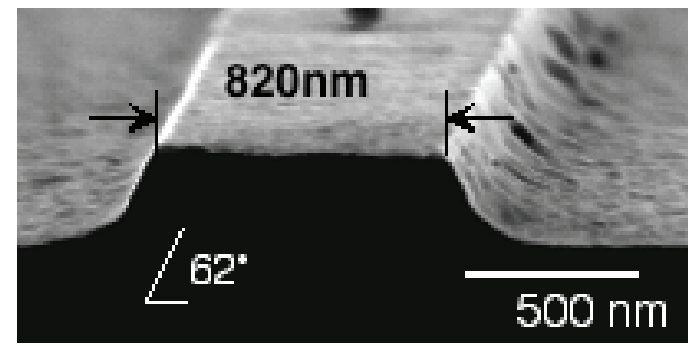

(a)

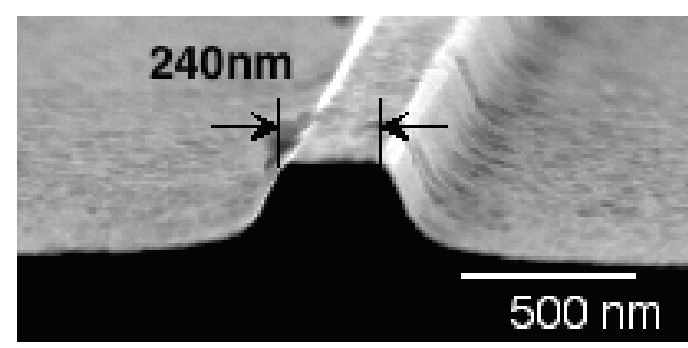

(b)

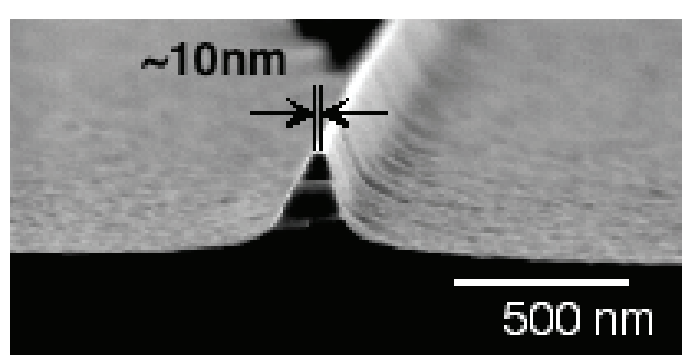

(c)

Fig. 6: Cross-sectional SEM images of samples after growth on $<11-20>$-oriented mesas having different pattern sizes of (a) $W_{0}=900 \mathrm{~nm}$, (b) $W_{0}=350 \mathrm{~nm}$ and (b) $W_{0}=50 \mathrm{~nm}$. 


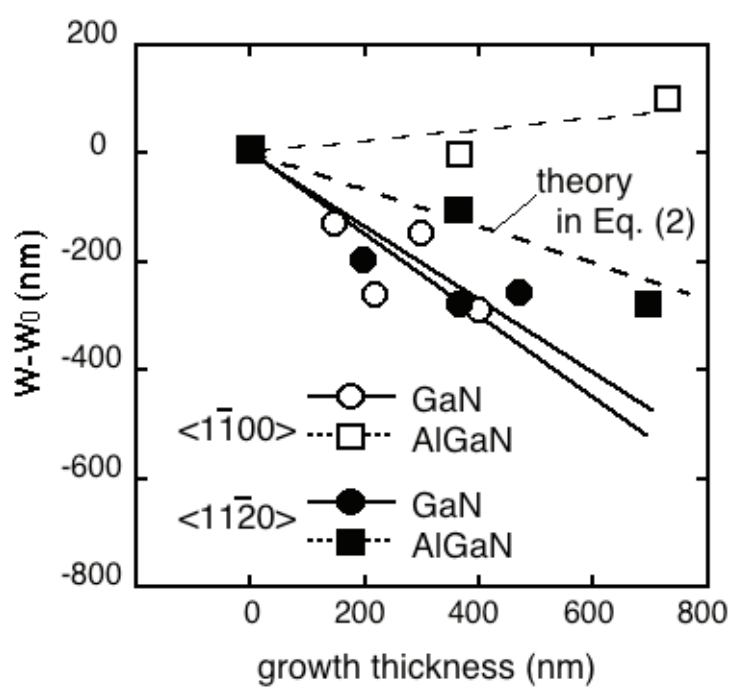

Fig. 7: Plot of top width of mesa vs. growth thickness.

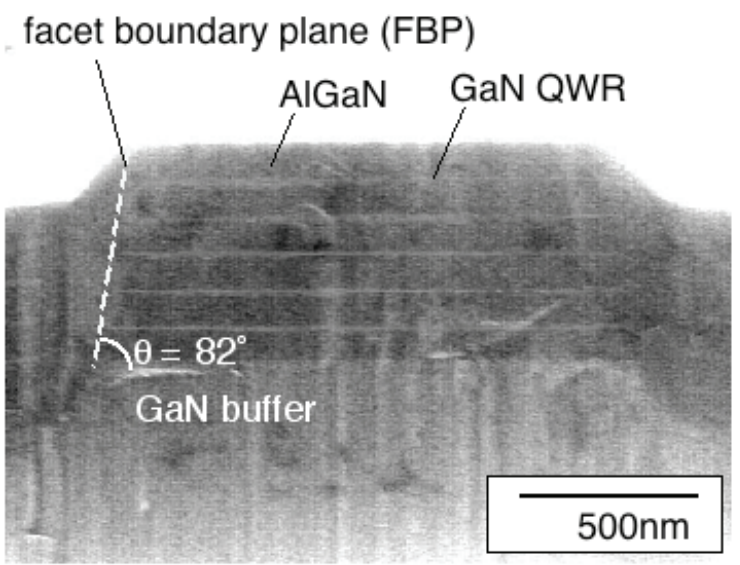

Fig. 8: Cross-sectional SEM images of GaN/AlGaN multilayer grown on $<11-20>$-oriented mesa.

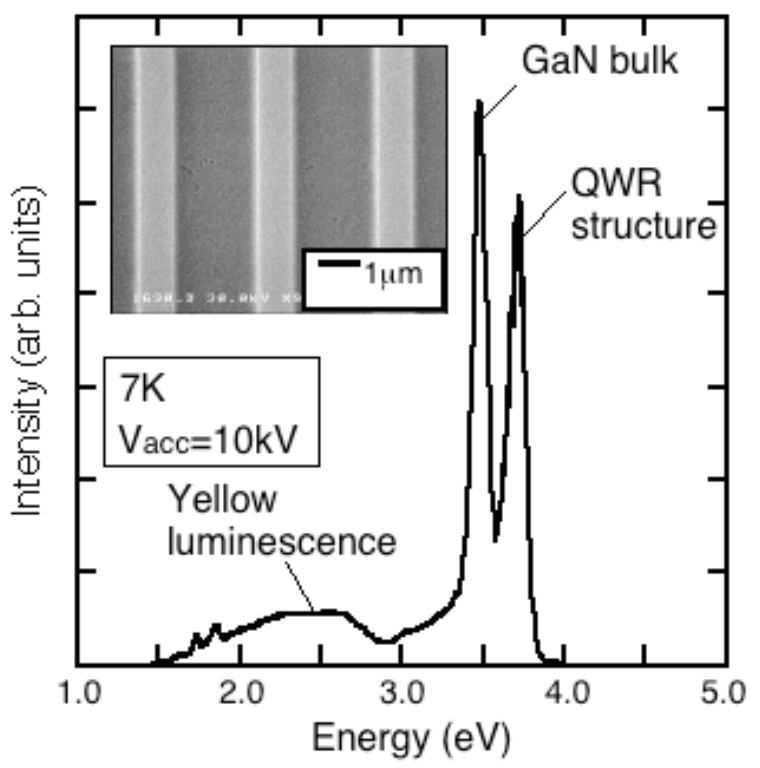

Fig. 9: CL spectra obtained from $<11-20>$-oriented QWR structure. 